(n)

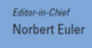
Journal of Nonlinear Mathematical Physics

\title{
Integrability Conditions for Complex Homogeneous Kukles Systems
}

Jaume Giné, Claudia Valls

To cite this article: Jaume Giné, Claudia Valls (2018) Integrability Conditions for Complex Homogeneous Kukles Systems, Journal of Nonlinear Mathematical Physics 25:3, 387398, DOI: https://doi.org/10.1080/14029251.2018.1494731

To link to this article: https://doi.org/10.1080/14029251.2018.1494731

Published online: 04 January 2021 


\title{
Integrability Conditions for Complex Homogeneous Kukles Systems
}

\author{
Jaume Giné \\ Departament de Matemàtica, Inspires Research Centre, Universitat de Lleida, \\ Avda. Jaume II, 69; 25001 Lleida, Catalonia, Spain \\ gine@matematica.udl.cat \\ Claudia Valls \\ Departamento de Matemática, Instituto Superior Técnico, \\ Av. Rovisco Pais 1049-001, Lisboa, Portugal \\ cvalls@math.ist.utl.pt
}

Received 20 December 2017

Accepted 12 February 2018

\begin{abstract}
In this paper we study the existence of local analytic first integrals for complex polynomial differential systems of the form $\dot{x}=x+P_{n}(x, y), \dot{y}=-y$, where $P_{n}(x, y)$ is a homogeneous polynomial of degree $n$, called the complex homogeneous Kukles systems of degree $n$. We characterize all the homogeneous Kukles systems of degree $n$ that belong to the Sibirsky ideal. Finally, we provide necessary and sufficient conditions when $n=$ $2, \ldots, 7$ in order that the complex homogeneous Kukles system has a local analytic first integral computing the saddle constants and using Gröbner bases to find the decomposition of the algebraic variety into its irreducible components.
\end{abstract}

Keywords: Integrability; complex center-focus problem; Saddle constants; Kukles systems; Gröbner Basis.

2010 Mathematics Subject Classification: 34C05, 37C10, 34C14

\section{Introduction and statement of the main results}

The integrability problem is one of the main problems in the qualitative theory of planar polynomial differential systems. Although integrability is a restrictive condition and a generic system is not integrable, the existence of a first integral allows to know the phase portrait of a planar differential system. These non-generic integrable differential systems are used to analyze a bigger family of differential systems that are described as perturbations of this non-generic system, see [1] and references therein. Closely related to the existence of a local analytic first integral is the so-called center-focus problem. The center problem for planar polynomial real systems of the form

$$
\dot{x}=-y+P(x, y), \quad \dot{y}=x+Q(x, y)
$$

being $P$ and $Q$ polynomials is equivalent to study when such systems admit a local analytic first integral of the form

$$
\Phi(x, y)=x^{2}+y^{2}+\sum_{j+k \geq 3} \phi_{j, k} x^{j} y^{k}, \quad \phi_{j, k} \in \mathbb{R} .
$$

In this case the trajectories are closed and the singular point at the origin is called a center. By definition any nonconstant differentiable function which is constant on the trajectories of (1.1) is a first integral of system (1.1). 
Setting $z=x+i y$ we can write system (1.1) of the form

$$
\dot{z}=i z+X(z, \bar{z})=i z+\sum_{k=2}^{n} X_{k}(z, \bar{z})
$$

where each $X_{k}$ is a homogeneous polynomial of degree $k$ and $\bar{z}=x-i y$. It turns easier and more convenient from the computational point of view to study the integrability problem not only for system (1.3) but also for more general complex systems

$$
\dot{z}=i z+\sum_{j+k=2}^{n} X_{j k} z^{j} \bar{z}^{k}, \quad \dot{\bar{z}}=-i \bar{z}+\sum_{j+k=2}^{n} Y_{j k} z^{j} \bar{z}^{k},
$$

where each $X_{j k}$ and $Y_{j k}$ are complex parameters. If we apply the change of time $t \mapsto i t$ system (1.4) becomes a system of the form

$$
\dot{z}=z-\sum_{j+k=2}^{n} a_{j k} z^{j} \bar{z}^{k}=z+P(z, \bar{z}), \quad \dot{\bar{z}}=-\bar{z}+\sum_{j+k=2}^{n} b_{j k} z^{j} \bar{z}^{k}=-\bar{z}+Q(z, \bar{z}) .
$$

For system (1.5) one can always find a function of the form

$$
\Phi(z, \bar{z})=z \bar{z}+\sum_{j+k \geq 3} \Phi_{j k} z^{j} \bar{z}^{k},
$$

such that

$$
\dot{\Phi}:=\frac{\partial \Phi}{\partial z}(z+P(z, \bar{z}))+\frac{\partial \Phi}{\partial \bar{z}}(-\bar{z}+Q(z, \bar{z}))=\sum_{s \geq 1} g_{s s}(z \bar{z})^{s+1}
$$

where $g_{s s}$ are polynomials of $a_{j k}$ and $b_{j k}$ with rational coefficients called saddle constants. In the case that $\dot{\Phi}=0$ we say that the origin is a complex center. Note that in this case $\Phi$ is a first integral of system (1.5). If the differential system (1.5) is a complexification of the differential system (1.1) then going back to the original coordinates we obtain from $\Phi$ a first integral of (1.1) of the form (1.2).

Conditions for the existence of a complex center for system (1.5) (that is for the existence of a first integral such as $\Phi$ in (1.6)) have been found for the case of quadratic systems $(n=2)$ (see for instance [4,6]). However, when $n \geq 3$ is an open and difficult problem, see [12]. The case $n=3$ with $P$ and $Q$ homogeneous polynomials was solved in [25]. The case when $P$ and $Q$ are of degree 3 but are not homogeneous was studied in [5] for a particular case.

In this paper we contribute to the characterization of the complex centers for a linear complex center perturbed by homogeneous polynomials of degree $n$, i.e., systems of the form

$$
\dot{x}=x-\sum_{j=-1}^{n-1} a_{j, n-1-j} x^{j+1} y^{n-1-j}, \quad \dot{y}=-y,
$$

where $x, y \in \mathbb{C}$ and $a_{j, n-1-j} \in \mathbb{C}$ not all zero since otherwise system (1.7) would be linear. Note that

$$
P_{n}(x, y):=\sum_{j=-1}^{n-1} a_{j, n-1-j} x^{j+1} y^{n-1-j}
$$

is a homogeneous polynomial of degree $n$. These systems are called complex Kukles homogeneous systems. The name comes from the fact that systems of this form in $\mathbb{R}^{2}$ and with the linear part with 
eigenvalues $\pm i$ were studied for the first time by Kukles in [18], who also was the first in studying a linear center perturbed by cubic nonhomogeneous nonlinearities. More specifically Kukles in [18] initiated the study of the necessary and sufficient conditions for the existence of a real center in differential systems of the form

$$
\begin{aligned}
& \dot{x}=y, \\
& \dot{y}=-x+a_{1} x^{2}+a_{2} x y+a_{3} y^{2}+a_{4} x^{3}+a_{5} x^{2} y+a_{6} x y^{2}+a_{7} y^{3},
\end{aligned}
$$

where $x, y \in \mathbb{R}$ and $a_{1}, \ldots, a_{7} \in \mathbb{R}$. The center problem for system (1.9) with $a_{2}=0$ was solved in [19] and with $a_{7}=0$ in [3]. The first complete solution of the center-focus problem for Kukles' system (1.9) was obtained in [20]. In [26] it was also given the complete solution using the Cherkas' method of passing to a Liénard equation, see also the works [2,3,10,17,21,27].

When $n=2$ or $n=3$ system (1.7) is a particular case of the system analyzed in [15], where the authors studied the system

$$
\begin{aligned}
& \dot{x}=x-a_{10} x^{2}-a_{01} x y-a_{12} y^{2}-a_{20} x^{3}-a_{11} x^{2} y-a_{02} x y^{2}-a_{13} y^{3}, \\
& \dot{y}=-y
\end{aligned}
$$

with $x, y \in \mathbb{C}$ and $a_{i j} \in \mathbb{C}$ not all zero. As a direct consequence of this study we state the following result without proof.

Theorem 1.1 ([15]). System (1.7) with $n=2$ has a complex center at the origin if and only if one of the following two conditions hold.

(a) $a_{1,0}=0$;

(b) $a_{0,1}=a_{-1,2}=0$.

System (1.7) with $n=3$ has a complex center at the origin if and only if one of the following two conditions hold.

(a) $a_{2,0}=a_{1,1}=0$;

(b) $a_{1,1}=a_{0,2}=a_{-1,3}=0$.

Before stating the main results of this paper we want to mention that the case when we have a real Kukles system with homogeneous nonlinearities, that is, a system of the form

$$
\dot{x}=y, \quad \dot{y}=-x+Q_{n}(x, y),
$$

where $x, y \in \mathbb{R}$ and $Q_{n}(x, y)$ a real homogeneous polynomial of degree $n$, was studied in [13] for the case $n$ odd and in [14] when $n$ is even. In particular, it is proved under certain conditions an open conjecture pointed in [28]: Is it true that a system (1.7) where $x, y \in \mathbb{R}$ with real nonlinearities of degree higher than two has a center at the origin if and only if its direction field is symmetric about one of the coordinate axes?

As we will see the case of complex centers is much more complicated to deal with than the real ones and the obtained results are much different from the ones regarding for real centers.

We introduce in Section 2 the subvariety called the Sibirsky subvariety of the center variety, see also $[16,22,23]$. The main results of the paper are the following.

Theorem 1.2. System (1.7) belongs to the Sibirsky variety if and only if one of the following two conditions hold: 
(a) when $n$ is even:

(a.1) $a_{n-1,0}=a_{n-2,1}=\cdots=a_{\frac{n}{2}, \frac{n}{2}-1}=0$
(a.2) $a_{\frac{n}{2}-1, \frac{n}{2}}=\cdots=a_{0, n-1}=a_{-1, n}=0$.

(b) when $n$ is odd:

(b.1) $a_{n-1,0}=a_{n-2,1}=\cdots=a_{\frac{n-1}{2}, \frac{n-1}{2}}=0$;

(b.2) $a_{\frac{n-1}{2}, \frac{n-1}{2}}=\cdots=a_{0, n-1}=a_{-1, n}^{2}=0$.

Theorem 1.2 is proved in section 3 . We recall that we have excluded from the Sibirsky variety when all coefficients $a_{i j}$ are zero since then system (1.7) would be the linear system that is not the case considered in this paper because we deal with nonlinear systems of degree $n$. Note that Theorem 1.2 is a general result for any $n \geq 2$. We recall that for every point in the Sibirsky subvariety the corresponding system has an analytic first integral defined in a neighborhood of the origin, see [16] and Theorem 3.5.5 of [24]. So systems provided by Theorem 1.2 have complex centers at the origin. We also recall that all time-reversible systems are inside the Sibirsky subvariety, see [22-24].

Now we characterize all the complex centers of system (1.7) when $n=4,5,6,7$. This is the content of the following four results.

Theorem 1.3. System (1.7) with $n=4$ has a complex center at the origin if and only if one of the following three conditions hold:

(a) $a_{3,0}=a_{2,1}=0$;

(b) $a_{1,2}=a_{0,3}=a_{-1,4}=0$;

(c) $a_{3,0}=a_{1,2}=a_{-1,4}=0$.

The proof of Theorem 1.3 is given in section 4 .

Theorem 1.4. System (1.7) with $n=5$ has a complex center at the origin if and only if one of the following three conditions hold:

(a) $a_{4,0}=a_{3,1}=a_{2,2}=0$;

(b) $a_{2,2}=a_{1,3}=a_{0,4}=a_{-1,5}=0$;

(c) $a_{4,0}=a_{2,2}=a_{1,3}=a_{-1,5}=0$.

The proof of Theorem 1.4 is given in section 5 .

Theorem 1.5. System (1.7) with $n=6$ has a complex center at the origin if and only if one of the following four conditions hold:

(a) $a_{5,0}=a_{4,1}=a_{3,2}=0$;

(b) $a_{2,3}=a_{1,4}=a_{0,5}=a_{-1,6}=0$;

(c) $a_{5,0}=a_{3,2}=a_{2,3}=a_{1,4}=a_{-1,6}=0$;

(d) $a_{5,0}=a_{4,1}=a_{2,3}=a_{1,4}=a_{-1,6}=0$.

The proof of Theorem 1.5 is given in section 6 .

Theorem 1.6. System (1.7) with $n=7$ has a complex center at the origin if and only if one of the following four conditions hold:

(a) $a_{6,0}=a_{5,1}=a_{4,2}=a_{3,3}=0$;

(b) $a_{3,3}=a_{2,4}=a_{1,5}=a_{0,6}=a_{-1,7}=0$; 
(c) $a_{6,0}=a_{5,1}=a_{3,3}=a_{2,4}=a_{1,5}=a_{-1,7}=0$;

(d) $a_{6,0}=a_{4,2}=a_{3,3}=a_{2,4}=a_{1,5}=a_{-1,7}=0$.

The proof of Theorem 1.6 is given in section 7 .

\section{The Sibirsky subvariety}

To state the main result of this paper we need to introduce the subvariety called the Sibirsky subvariety of the center variety. To define this subvariety we proceed as follows. We consider a system of the form

$$
\dot{x}=x-\sum_{(p, q) \in S} a_{p q} x^{p+1} y^{q}, \quad \dot{y}=-y+\sum_{(p, q) \in S} b_{q p} x^{q} y^{p+1},
$$

where $a_{p q}, b_{q p} \in \mathbb{C}$ and

$$
S=\left\{\left(p_{j}, q_{j}\right) \mid p_{j}+q_{j} \geq 1, j=1, \ldots, l\right\} \subset\{\{-1\} \cup \mathbb{N}\} \times \mathbb{N} .
$$

Let $L: \mathbb{N}^{2 l} \rightarrow \mathbb{N}^{2}$ be the linear operator such that for $v=\left(v_{1}, \ldots, v_{2 l}\right) \in \mathbb{N}^{2 l}$,

$$
L(v):=\left(\begin{array}{c}
p_{1} \\
q_{1}
\end{array}\right) v_{1}+\cdots+\left(\begin{array}{c}
p_{l} \\
q_{l}
\end{array}\right) v_{l}+\left(\begin{array}{c}
q_{l} \\
p_{l}
\end{array}\right) v_{l+1}+\cdots+\left(\begin{array}{c}
q_{1} \\
p_{1}
\end{array}\right) v_{2 l}=\left(\begin{array}{c}
L_{1}(v) \\
L_{2}(v)
\end{array}\right)
$$

and let

$$
\mathscr{M}:=\bigcup_{k \in \mathbb{N}}\left\{v \in \mathbb{N}^{2 l}: L(v)=\left(\begin{array}{l}
k \\
k
\end{array}\right)\right\}
$$

Let also $\mathbb{C}[\mathscr{M}]$ denote the subalgebra of $\mathbb{C}[a, b]$ generated by all monomials of the form

$$
a_{p_{1} q_{1}}^{v_{1}} a_{p_{2} q_{2}}^{v_{2}} \cdots a_{p_{l} q_{l}}^{v_{l}} b_{q_{l} p_{l}}^{v_{l+1}} b_{q_{l-1} p_{l-1}}^{v_{l+2}} \cdots b_{q_{1} p_{1}}^{v_{2 l}}
$$

for all $v \in \mathscr{M}$. In order to simplify notation we will abbreviate such a monomial by $[v]=$ $\left[v_{1}, \ldots, v_{2 l}\right]$. For $v \in \mathscr{M}$, let

$$
\bar{v}=\left(v_{2 l}, \ldots, v_{1}\right) .
$$

The Sibirsky ideal is

$$
I_{\mathrm{sib}}=\langle[v]-[\bar{v}] \mid v \in \mathscr{M}\rangle .
$$

As pointed out in the introduction, this variety is very important since for every point in the Sibirsky subvariety the corresponding system has an analytic first integral defined in a neighborhood of the origin, see [16].

For system (1.7), for any fixed $n$, we have that $l=n+1$, and $\left(p_{1}, q_{1}\right)=(0, n-1),\left(p_{2}, q_{2}\right)=$ $(1, n-2), \ldots,\left(p_{n+1}, q_{n+1}\right)=(-1, n)$. Moreover, since $\mathbb{C}[\mathscr{M}]$ denote the subalgebra of $\mathbb{C}[a, b]$ generated by all monomials of the form (2.2) and for system (1.7) we have that $b_{q_{j} p_{j}}=0$ for $j=1, \ldots, l=n+1$, in $\mathscr{M}$ we can take

$$
v_{l+1}=\cdots=v_{2 l}=v_{n+2}=\cdots=v_{2(n+1)}=0
$$

since otherwise $\mathbb{C}[\mathscr{M}]=\{0\}$ and this case is not considered here since this correspond to the linear systems which are not taken into account due to the fact that we deal with nonlinear systems of degree $n \geq 2$. 


\section{Proof of Theorem 1.2}

Note that in system (2.1) we have $b_{q p}=0$ for all $p, q \in S$ and (2.1) can be written in the form of system (1.7).

Lemma 3.1. The Sibirsky component of the center variety of system (1.7) for each fixed $n \geq 2$ is defined by the following equations

$$
\prod_{j=0}^{n-1} a_{j, n-1-j}^{v_{j+1}} a_{-1, n}^{v_{n+1}}=0
$$

with $v_{j} \in \mathbb{N}$ for $j=-1, \ldots, n-1$ such that

$$
\sum_{j=1}^{n}(n-2 j+1) v_{j}+(n+1) v_{n+1}=0 .
$$

Proof. The proof follows directly from the definition of Sibirsky variety taking into account that in our case $b_{p q}=0$ for all $p, q \in S$ and so $v=\left(v_{1}, \ldots, v_{n}, v_{n+1}\right)$ (see the last paragraph in section 2). Hence the condition defining the Sibirsky variety becomes

$$
\left(\begin{array}{c}
0 \\
n-1
\end{array}\right) v_{1}+\left(\begin{array}{c}
1 \\
n-2
\end{array}\right) v_{2}+\cdots+\left(\begin{array}{c}
n-1 \\
0
\end{array}\right) v_{n}+\left(\begin{array}{c}
-1 \\
n
\end{array}\right) v_{n+1}=\left(\begin{array}{l}
k \\
k
\end{array}\right)
$$

or in other words

$$
\begin{aligned}
v_{2}+2 v_{3}+\cdots+(n-1) v_{n}-v_{n+1} & =k, \\
(n-1) v_{1}+(n-2) v_{2}+\cdots+v_{n-1}+n v_{n+1} & =k,
\end{aligned}
$$

which yields

$$
\begin{aligned}
& v_{2}+2 v_{3}+\cdots+(n-1) v_{n}-v_{n+1} \\
& =(n-1) v_{1}+(n-2) v_{2}+\cdots+v_{n-1}+n v_{n+1}
\end{aligned}
$$

This condition can be written as

$$
(n-1) v_{1}+(n-3) v_{2}+\cdots+(1-(n-2)) v_{n-1}-(n-1) v_{n}+(n+1) v_{n+1}=0,
$$

or equivalently

$$
\sum_{j=1}^{n}(n-2 j+1) v_{j}+(n+1) v_{n+1}=0 .
$$

This concludes the proof. 
Proof. [Proof of Theorem 1.2] We first take $n \geq 2$ even. In this case we can write (3.1) as follows

$$
\sum_{j=1}^{n / 2}(n-2 j+1) v_{j}+(n+1) v_{n+1}-\sum_{j=n / 2+1}^{n}(2 j-1-n) v_{j}=0
$$

that is

$$
v_{\frac{n}{2}}+\sum_{j=1}^{n / 2-1}(n-2 j+1) v_{j}+(n+1) v_{n+1}=v_{\frac{n}{2}+1}+\sum_{j=n / 2+2}^{n}(2 j-1-n) v_{j}
$$

Since $v_{j} \in \mathbb{N}$, and $v_{j}$ is the exponent of $a_{j-1, n-j}$ for $j=1, \ldots, n$ and $v_{n+1}$ is the exponent of $a_{-1, n}$, it is clear that the two conditions are

$$
a_{\frac{n}{2}-1, \frac{n}{2}}=\cdots=a_{0, n-1}=a_{-1, n}=0
$$

and

$$
a_{\frac{n}{2}, \frac{n}{2}-1}=\cdots=a_{n-2,1}=a_{n-1,0}=0 .
$$

This concludes the proof when $n$ is even.

When $n$ is odd, it follows from the general theory of Lyapunov constants that $a_{\frac{n-1}{2}}, \frac{n-1}{2}=0$ since it corresponds to the first Lyapunov constant.

In this case we can write (3.1) as follows

$$
\sum_{j=1}^{(n-1) / 2}(n-2 j+1) v_{j}+(n+1) v_{n+1}-\sum_{j=(n+3) / 2}^{n}(2 j-1-n) v_{j}=0,
$$

that is

$$
v_{\frac{n-1}{2}}+\frac{1}{2} \sum_{j=1}^{(n-3) / 2}(n-2 j+1) v_{j}+(n+1) v_{n+1}=v_{\frac{n+3}{2}}+\frac{1}{2} \sum_{j=(n+5) / 2}^{n}(2 j-1-n) v_{j}
$$

Since $v_{j} \in \mathbb{N}$, and $v_{j}$ is the exponent of $a_{j-1, n-j}$ for $j=1, \ldots, n$ and $v_{n+1}$ is the exponent of $a_{-1, n}$, it is clear that the two conditions are

$$
a_{\frac{n-1}{2}, \frac{n-1}{2}}=a_{\frac{n-3}{2}, \frac{n+1}{2}} \cdots=a_{0, n-1}=a_{-1, n}=0
$$

and

$$
a_{\frac{n-1}{2}, \frac{n-1}{2}}=a_{\frac{n+1}{2}, \frac{n-3}{2}}=\cdots=a_{n-2,1}=a_{n-1,0}=0 .
$$

This concludes the proof.

Before giving the proof of Theorem 1.3 we give the following result proved in [4]. If an integrating factor is not well defined in a neighborhood of a singular point the following statement can be applied. 
Lemma 3.2. (i) If system

$$
\dot{x}=x-\sum_{p+q=1}^{n-1} a_{p, q} x^{p+1} y^{q}, \quad \dot{y}=-y+\sum_{p+q=1}^{n-1} b_{q, p} x^{q} y^{p+1}
$$

has a local inverse integrating factor $\mu(x, y)=(x y)^{\alpha} \prod_{i=1}^{m} F_{i}^{\beta_{i}}$ with $F_{i}$ analytic in $x$ and $y, F_{i}(0,0)=0$ for $i=1, \ldots, m, \alpha \neq 0$, and $\alpha$ is not an integer greater than 1 , then it has a first integral of the form (1.6).

(ii) If system (3.2) has a local inverse integrating factor $\mu(x, y)=(x y)^{\alpha}$ and $a_{\alpha, \alpha-1}=b_{\alpha-1, \alpha}=0$, then it has a first integral of the form (1.6).

\section{Proof of Theorem 1.3}

We first establish the sufficiency of all conditions of Theorem 1.3. Statements (a) and (b) are the Sibirsky cases given in Theorem 1.2 when $n=4$. For statement (c) system (1.7) becomes

$$
\begin{aligned}
& \dot{x}=x-a_{2,1} x^{3} y-a_{0,3} x y^{3} \\
& \dot{y}=-y .
\end{aligned}
$$

This system has two invariant lines $l_{1}=x, l_{2}=y$, and one exponential factor $f_{1}=e^{y^{3}}$. Moreover it is possible to find an inverse integrating factor is of the form $V=\left(l_{1} l_{2}\right)^{3} e^{-2 a_{0,3} y^{3} / 3}$. In order to prove the existence of an analytic first integral in a neighborhood of the origin we apply statement (ii) of Lemma 3.2, First we make a change of time given by $d \tau=e^{-2 a_{0,3} y^{3} / 3} d t$. The new system has an inverse integrating factor $V=(x y)^{3}$ and we can apply statement (ii) of Lemma 3.2 to deduce the existence of an analytic first integral of the form (1.6).

Now we prove the necessity of all conditions of Theorem 1.3. For doing this, we introduce in system (1.7) with $n=4$ the change of variables $X=x+i y, Y=x-i y$ and $t \mapsto t / i$. With this change, (1.7) becomes

$$
\begin{aligned}
\dot{X} & =Y+i\left(a_{03}+a_{12}+a_{14}+a_{21}+a_{30}\right) X^{4} / 2 \\
& +\left(a_{03}+2 a_{14}-a_{21}-2 a_{30}\right) X^{3} Y+i\left(a_{12}-3\left(a_{14}+a_{30}\right)\right) X^{2} Y^{2} \\
& +\left(a_{03}-2 a_{14}-a_{21}+2 a_{30}\right) X Y^{3}-i\left(a_{03}-a_{12}-a_{14}+a_{21}-a_{30}\right) Y^{4} / 2, \\
\dot{Y} & =-X+\left(a_{03}+a_{12}+a_{14}+a_{21}+a_{30}\right) X^{4} / 2 \\
& -i\left(a_{03}+2 a_{14}-a_{21}-2 a_{30}\right) X^{3} Y+\left(a_{12}-3\left(a_{14}+a_{30}\right)\right) X^{2} Y^{2} \\
& -i\left(a_{03}-2 a_{14}-a_{21}+2 a_{30}\right) X Y^{3}-\left(a_{03}-a_{12}-a_{14}+a_{21}-a_{30}\right) Y^{4} / 2 .
\end{aligned}
$$

Now we write (4.1) in polar coordinates, i.e., we do the change of variables $r^{2}=z \bar{z}$ and $\theta=$ $\arctan (\operatorname{Im} z / \operatorname{Re} z)$, and system (4.1) becomes

$$
\begin{aligned}
\dot{r}= & i r^{4}\left(\left(a_{12}+a_{21}\right) \cos \theta+\left(a_{03}+a_{30}\right) \cos 3 \theta+a_{14} \cos 5 \theta\right. \\
& \left.+i\left(a_{21}-a_{12}\right) \sin \theta+i\left(a_{30}+a_{03}\right) \sin 3 \theta-i a_{14} \sin 5 \theta\right) / 2 \\
\dot{\theta}= & -1+r^{3}\left(\left(a_{12}+a_{21}\right) \cos \theta+\left(a_{03}+a_{30}\right) \cos 3 \theta+a_{14} \cos 5 \theta\right. \\
& \left.+i\left(a_{21}-a_{12}\right) \sin \theta+i\left(a_{30}-a_{03}\right) \sin 3 \theta-i a_{14} \sin 5 \theta\right) / 2 .
\end{aligned}
$$

To determine the necessary conditions to have a center for system (4.2) we propose a formal Poincaré series of the form

$$
H(r, \theta)=\sum_{n=2}^{\infty} H_{n}(\theta) r^{n}
$$


where $H_{2}(\theta)=1 / 2$ and $H_{n}(\theta)$ are homogeneous trigonometric polynomials respect to $\theta$ of degree $n$. Imposing that this power series is a formal first integral of system (4.2) we obtain

$$
\dot{H}(r, \theta)=\sum_{k=2}^{\infty} V_{2 k} r^{2 k}
$$

where $V_{2 k}$ are in fact the Poincaré-Liapunov constants that depend on the parameters of system (4.1) which correspond to the saddle (focus) quantities of the original system (1.7) with $n=4$. It is easy to see that these $V_{2 k}$ are polynomials in the parameters of system (4.1). System (4.1) is polynomial and due to the Hilbert Basis theorem, the ideal $J=\left\langle V_{2}, V_{4}, \ldots\right\rangle$ generated by the Poincaré-Liapunov constants is finitely generated, i.e. there exist $W_{1}, W_{2}, \ldots, W_{k}$ in $J$ such that $J=\left\langle W_{1}, W_{2}, \ldots, W_{k}\right\rangle$. Such a set of generators is called a basis of $J$ and the conditions $W_{j}=0$ for $j=1, \ldots, k$ provide a finite set of necessary conditions to have a center. The set of coefficients for which all the saddle constants $V_{2 k}$ vanish is called the complex center variety of system (1.7) with $n=4$ and it is an algebraic set.

In practice we determine a finite number of Poincaré-Liapunov constants thinking that inside these number there is the set of generators. From this set a much harder problem is to decompose this algebraic set into its irreducible components. We must use a computer algebra system. The computational tool which we use is the routine minAssGTz [7] of the computer algebra system SINGULAR [9] which is based on the Gianni-Trager-Zacharias algorithm [8]. The computations have been completed in the field of rational numbers. That is, we know that the decomposition of the center variety that we encountered is complete. Therefore in the statement of the theorem we provide sufficient and necessary conditions to have a complex center.

\section{Proof of Theorem 1.4}

We first establish the sufficiency of all conditions of Theorem 1.4. Statements (a) and (b) are the Sibirsky cases given in Theorem 1.2 when $n=5$. For statement (c) system (1.7) becomes

$$
\begin{aligned}
& \dot{x}=x-a_{3,1} x^{4} y-a_{0,4} x y^{4} \\
& \dot{y}=-y .
\end{aligned}
$$

This system has two invariant lines $l_{1}=x, l_{2}=y$, and one exponential factor $f_{1}=e^{y^{4}}$. With these ingredients we can find an inverse integrating factor of the form $V=\left(l_{1} l_{2}\right)^{4} e^{-2 a_{0,4 y^{3}} / 3}$. To prove the existence of an analytic first integral in a neighborhood of the origin we also apply statement (ii) of Lemma 3.2. We make a change of time given by $d \tau=e^{-2 a_{0,4 y^{3}} / 3} d t$. The new system has an inverse integrating factor $V=(x y)^{4}$ and we can apply statement (ii) of Lemma 3.2 to deduce the existence of an analytic first integral of the form (1.6).

The necessity of all conditions of Theorem 1.4 is proved by the same method used in the previous theorem.

\section{Proof of Theorem 1.5}

The sufficiency of all conditions of statements (a) and (b) of Theorem 1.5 come from Theorem 1.2 when $n=6$. For statement (c), note that system (1.7) becomes

$$
\begin{aligned}
& \dot{x}=x-a_{4,1} x^{5} y-a_{0,5} x y^{5} \\
& \dot{y}=-y .
\end{aligned}
$$


System (6.1) has two invariant lines $l_{1}=x, l_{2}=y$, one exponential factor $f_{1}=e^{y^{5}}$ and an inverse integrating factor of the form $V=\left(l_{1} l_{2}\right)^{5} e^{-4 a_{0,5} y^{5} / 5}$. To prove the existence of an analytic first integral in a neighborhood of the origin we apply statement (ii) of Lemma 3.2. We made a change of time given by $d \tau=e^{-4 a_{0,4 y^{3}} / 5} d t$. The new system has an inverse integrating factor $V=(x y)^{5}$ and we can apply statement (ii) of Lemma 3.2 to deduce the existence of an analytic first integral of the form (1.6).

For statement (d), note that system (1.7) becomes

$$
\begin{aligned}
& \dot{x}=x-a_{3,2} x^{4} y^{2}-a_{0,5} x y^{5} \\
& \dot{y}=-y .
\end{aligned}
$$

System (6.2) has two invariant lines $l_{1}=x, l_{2}=y$, one exponential factor $f_{1}=e^{y^{4}}$ and an inverse integrating factor of the form $V=\left(l_{1} l_{2}\right)^{4} e^{-3 a_{0,5} y^{5} / 4}$. To prove the existence of an analytic first integral in a neighborhood of the origin we also apply statement (ii) of Lemma 3.2 by making a change of time similarly as in the proof of Theorem 1.4.

The necessity of conditions of Theorem 1.5 is proved by the same method used in Theorem 1.3.

\section{Proof of Theorem 1.6}

The sufficiency of all conditions of statements (a) and (b) of Theorem 1.6 come from Theorem 1.2 when $n=7$. For statement (c), note that system (1.7) becomes

$$
\begin{aligned}
& \dot{x}=x-a_{4,2} x^{5} y^{2}-a_{-1,6} x y^{5} \\
& \dot{y}=-y .
\end{aligned}
$$

System (7.1) has two invariant lines $l_{1}=x, l_{2}=y$, one exponential factor $f_{1}=e^{y^{6}}$ and an inverse integrating factor of the form $V=\left(l_{1} l_{2}\right)^{5} e^{-4 a_{-1,6} y^{6} / 6}$.

For statement (d), note that system (1.7) becomes

$$
\begin{aligned}
& \dot{x}=x-a_{4,1} x^{5} y-a_{-1,6} x y^{5} \\
& \dot{y}=-y .
\end{aligned}
$$

System (7.2) has two invariant lines $l_{1}=x, l_{2}=y$, one exponential factor $f_{1}=e^{y^{4}}$ and an inverse integrating factor of the form $V=\left(l_{1} l_{2}\right)^{6} e^{-5 a_{-1,6} y^{6} / 6}$. To prove the existence of an analytic first integral in a neighborhood of the origin in both cases we apply statement (ii) of Lemma 3.2 in the same way that in the previous cases, by making a convenient change of time.

The necessity of conditions of Theorem 1.6 is proved by the same method used in Theorem 1.3.

In view of the results obtained we can formulate the following conjecture: Is it true that a system (1.7) has a complex center at the origin if and only if it belongs to the Sibirsky ideal or it is Liouville integrable?

Recall that a differential system in the plane is Liouville integrable if it has an integrating factor of the form $V=\exp \{D / E\} \prod_{i} C_{i}^{\lambda_{i}}$ where $D, E$ and $C_{i} \in \mathbb{C}[x, y]$ and $\lambda_{i} \in \mathbb{C}$. Note that $E$ and $C_{i}$ are invariant algebraic curves of the system and $\exp \{D / E\}$ is an exponential factor, see for instance [11] and references therein. 


\section{Acknowledgements}

The first author is partially supported by a MINECO/ FEDER grant number 2017-84383-P and an AGAUR (Generalitat de Catalunya) grant number 2017SGR 1276. The second author is partially supported by FCT/Portugal through UID/MAT/04459/2013.

\section{References}

[1] A. Algaba, C. García, J. Giné, On the formal integrability problem for planar differential systems, Abtract and Applied Analysis 2013 (2013) ID482305 10 pp.

[2] L.A. Cherkas, Conditions for the equation $y y^{\prime}=\sum_{i=0}^{3} p_{i}(x) y^{i}$ to have a centre, Differential Equations 14 (1978) 1133-1138.

[3] C.J. Christopher, N.G. Lloyd, On the paper of Jin and Wang concerning the conditions for a centre in certain cubic systems, Bull. London Math. Soc. 22 (1990) 5-12.

[4] C. Christopher, P. Mardešić, C. Rousseau, Normalizable, integrable and linearizable saddle points for complex quadratic systems in $\mathbb{C}^{2}$, J. Dyn. Control Syst. 9 (2003) 311-363.

[5] M. Dukarić, J. Giné, Integrability of Lotka-Volterra planar complex cubic systems, Internat. J. Bifur. Chaos Appl. Sci. Engrg. 26, no. 1, (2016), 1650002, 16 pp.

[6] H. Dulac, Détermination et intégration d'une certain classe d'équations différentielles ayant pour point singulier un centre, Bull. Sci. Math. 32 (1980) 230-252.

[7] W. Decker, S.Laplagne, G. Pfister, H.A. Schonemann, SINGULAR 3-1 library for computing the prime decomposition and radical of ideals, primdec.lib, 2010.

[8] P. Gianni, B. Trager, G. Zacharias, Gröbner bases and primary decompositions of polynomials, J. Symbolic Comput. 6 (1988) 146-167.

[9] G.M. Greuel, G. Pfister, H.A. Schönemann, SInGULAR 3.0 A Computer Algebra System for Polynomial Computations, Centre for Computer Algebra, University of Kaiserlautern (2005). http://www . singular.uni-kl.de.

[10] J. Giné, Conditions for the existence of a center for the Kukles homogenenous systems, Comput. Math. Appl. 43 (2002) 1261-1269.

[11] J. Giné, Reduction of integrable planar polynomial differential systems, Appl. Math. Lett. 25 (2012), no. $11,1862-1865$.

[12] J. Giné, V.G. Romanovski, Integrability conditions for Lotka-Volterra planar complex quintic systems, Nonlinear Anal. Real World Appl. 11 (2010) 2100-2105.

[13] J. Giné, J. Llibre, C. Valls, Centers for the Kukles homogeneous systems with odd degree, Bull. London Math. Soc. 47 (2015) 315-324.

[14] J. Giné, J. Llibre, C. Valls, Centers for the Kukles homogeneous systems with even degree, J. Appl. Anal. Comput. 7 (2017), no. 4, 1534-1548.

[15] J. Giné, C. Valls, Integrability conditions for complex Kukles systems, Dynamical Systems 32 (2017), no. 2, 211-220.

[16] A.S. Jarrah, R. Laubenbacher, V. Romanovski, The Sibirsky component of the center variety of a polynomial differential systems, J. Symbolic Comput. 35 (2003), 577-589.

[17] X. Jin, D. Wang, On the conditions of Kukles for the existence of a centre, Bull. London Math. Soc. 22 (1990), $1-4$.

[18] I.S. Kukles, Sur quelques cas de distinction entre un foyer et un centre, Dokl. Akad. Nauk. SSSR 42 (1944) 208-211.

[19] N.G. Lloyd, J.M. Pearson, Conditions for a centre and the bifurcation of limit cycles, in: J.-P. Françoise, R. Roussarie (Eds.), Bifurcations of Planar Vector Fields, Lect. Notes. in Math. 1455 (Springer, 1990) 230-242.

[20] N.G. Lloyd, J.M. Pearson, Computing centre conditions for certain cubic systems, J. Comp. Appl. Math. 40 (1992) 323-336.

[21] J.M. Pearson, N.G. Lloyd, Kukles revisited: advances in computing techniques, Comput. Math. Appl. 60 (2010), no. 10, 2797-2805. 
[22] V.G. Romanovski, Time-Reversibility in 2-Dimensional Systems, Open Syst. Inf. Dynamics 15 (2008) 359-370.

[23] V.G. Romanovski, D.S. Shafer, Time-reversibility in two-dimensional polynomial systems, in: Trends in Mathematics, Differential Equations with Symbolic Computations (D. Wang and Z. Zheng, Eds.), 67-84 (Birkhäuser-Verlag, Basel, 2005).

[24] V.G. Romanovski, D.S. Shafer, The center and cyclicity problems: a computational algebra approach, (Birkhäuser Boston, Inc., Boston, MA, 2009).

[25] A.P. Sadovskii, Holomorphic integrals of a certain system of differential equations, Differ. Uravn. 10 (1974) 558-560; Differ. Equ. 10 (1974) 425-427.

[26] A.P. Sadovskii, Solution of the center and focus problem for a cubic system of nonlinear oscillations, Differ. Uravn. 33 (1997), no. 2, 236-244, 286; Differential Equations 33 (1997), no. 2, 236-244.

[27] A.S. Shubè, On the Kukles and Cherkas center conditions for a cubic system, Differential Equations 29 (1993) 625-627.

[28] E.P. Volokitin, V.V. Ivanov, Isochronicity and Commutation of polynomial vector fields, Siberian Mathematical Journal 40 (1999) 22-37. 